\title{
Statistical Analysis of Field-Emission Currents
}

\author{
Sagy Lachmann, ${ }^{1}$ Marek Jacewicz $\odot,{ }^{2}$ Iaroslava Profatilova $\odot,{ }^{3}$ Jan Paszkiewicz, ${ }^{3}$ Walter Wuensch $\odot,{ }^{3}$ \\ and Yinon Ashkenazy ${ }^{1, *}$ \\ ${ }^{1}$ Racah Institute of Physics, Hebrew University of Jerusalem, Edmond J. Safra Campus, 9190401 Jerusalem, Israel \\ ${ }^{2}$ Department of Physics and Astronomy, Uppsala University, Regementsvägen 1, SE-752 37 Uppsala, Sweden \\ ${ }^{3}$ CERN, European Organization for Nuclear Research, 1211 Geneva, Switzerland
}

(Received 21 April 2021; revised 27 June 2021; accepted 19 July 2021; published 4 August 2021)

\begin{abstract}
The current due to cold tunneling of electrons from a metallic surface exposed to high electric fields, regularly named "dark current," is commonly described in modern literature using an analytical approximate solution provided by Murphy and Good [Phys. Rev. 102, 1464 (1956)]. This expression, which corrects earlier work by Fowler and Nordheim, is a Fowler-Nordheim-type equation: $I \sim E^{2} \exp (-a / E)$, where $I$ is the dark current, $E$ is the local electric field, and $a$ is a system-specific constant. In this paper, a numerical approximation, rather than the analytical one given by Murphy and Good, is presented. This approximation is accurate over a wide range of fields, and is used to derive the effective field enhancement factor $\beta$. On the basis of this approximation, and considering local field and current fluctuations, two alternative methods for $\beta$ estimation are presented. These methods allow instantaneous field-specific estimation of $\beta$, rather than the average estimate derived with current methods. The applicability of fluctuation-based methods is demonstrated by numerical simulation in a variety of conditions. The methods are applied to estimate $\beta$ using fluctuation analysis in experimental measurements that were not dedicated for this purpose. An open-source code for the implementation of fluctuation-derived $\beta$ estimation is provided, with an analysis of possible future experimental opportunities using dedicated experiments.
\end{abstract}

DOI: 10.1103/PhysRevApplied.16.024007

\section{INTRODUCTION}

Dark current due to tunneling of electrons between electrodes separated by a vacuum under high electric fields is a central part of models for electric arc formation in a vacuum, also known as vacuum breakdown (BD). It is assumed that field emission is a critical step of the multistep process leading to BD [1-4], and an increase in current, which leads to localized melting during arc formation, was previously observed [5-7]. However there are only initial indications of a link between the current before BD and BD formation [8]. Dark currents can also be problematic in and of themselves, disrupting acceleratingbeam instrumentation, for example, resulting in a loss of performance in certain high-gradient systems [9].

The dark currents preceding BD formation are measurable, and their mean dependence on the applied field is well

*yinon.ash@mail.huji.ac.il

Published by the American Physical Society under the terms of the Creative Commons Attribution 4.0 International license. Further distribution of this work must maintain attribution to the author(s) and the published article's title, journal citation, and DOI. reproduced by a simple analysis relying on conductionelectron tunneling to the vacuum. Modern BD literature uses the Murphy-Good (MG) equation [10] (for a detailed derivation see Ref. [11]), which corrected the original treatment of this problem by Fowler and Nordheim, known as the Fowler-Nordheim (FN) equation [12]. The MG equation describes the mean emitted current due to cold electron tunneling and has been regularly used in a wide range of studies [6,7,13-15]. The MG current equation contains two unknown parameters: the effective emitting area and the field enhancement factor, denoted as $\beta$. "Field enhancement" refers to the ratio between the macroscopic applied field and the microscopic local field calculated at the current emission site, so a larger $\beta$ yields higher currents. While the area dependence affects the absolute value of the current linearly, $\beta$ affects its relative variation in the field, and thus it is extremely common to characterize systems exposed to high electric fields using a semilogarithmic plot of the current $-\log _{10}\left(I / E_{0}^{2}\right)$ as a function of $1 / E_{0}$ — called a "Fowler-Nordheim plot" [7].

Previous experimental observations of dark currents in exposed metallic electrodes in a vacuum, reached maximum applied electric fields of 40-250 MV/m, which, taking into account the estimated field enhancement factors, lead to a localized field at the emission site of $7.5-15 \mathrm{GV} / \mathrm{m}$ 
[1,16-19]. Thus, the range of local fields $\left(\beta E_{0}\right)$ we explore in this paper is $5-15 \mathrm{GV} / \mathrm{m}$.

In this paper, two methods of $\beta$ estimation are presented along with numerical adjustments to the current $\beta$-analysis method as described by Wang and Loew [7]. These methods rely on statistical analysis of the current distribution with time, compared with a mean-current analysis with the FN-plot-based method. These methods allow instantaneous field-dependent estimation of $\beta$, which could help explore pre-BD dynamics and their effect on the measured current. Here, we retain the standard assumption that the measured dark current can be described as being controlled by an amplified field with a single fixed factor described by $\beta[7,10,12]$.

It is important to note at this point that the theory presented in Sec. II [mainly Eqs. (2), (3), and (5)] has since been expanded [20-22] and can still be refined further. This paper focuses on this version of the current equation (as presented in Refs. [7,10,22,23] and as detailed in Sec. II) for a direct comparison with the Wang-Loew $\beta$ estimation method [7]. The methods presented in this paper can therefore also be refined further and improved to better describe physical systems by accounting for additional physical effect (e.g., as discussed in Refs. [20-22]). This paper demonstrates the benefits of using statistical analysis of dark currents - both as improvement of estimation errors and as adding the capability of field-specific $\beta$ estimation. This is demonstrated for a specific range of fields and under a set of assumptions, which could be extended and improved in future work.

\section{APPROXIMATION OF THE DARK-CURRENT EXPRESSION}

A broadly accepted equation describing the dark current from a field emitter was developed by Murphy and Good $[10,11]$, based on the original model by Fowler and Nordheim [12]. In this model, the dark current $I$ is expressed in terms of the electronic supply function $N(W)$ and the transmission (tunneling) probability $D(W)$, where $W$ is the electron energy normal to the barrier measured relative to the base of the conduction band, leading to

$$
I=S e \int D(W) N(W) d W
$$

with $S$ being an effective current-emitting surface area. We assume that the current created from the multiple current emitters expected to be found on macroscopic cathodes can be described effectively using a single "average" emitter characterized by an effective enhanced field $\beta E_{0}$ and $S$.

$N(W)$, the electronic supply function, is given by

$$
N(W)=\frac{4 \pi m k_{B} T}{h^{3}} L\left(\frac{W-\mu}{k T}\right),
$$

where $L(x)=\ln [1+\exp (-x)]$, and $D(W)$, the onedimensional transmission probability, is given by

$$
D(W)=\left\{1+\exp \left[B H^{3 / 2} v(y) E^{-1}\right]\right\}^{-1},
$$

where $H$ is the zero-field barrier height (i.e., the energy difference between the local vacuum level and the normalenergy level of the electron $W$ ), $T$ is the temperature, and $E=\beta E_{0}$ is the local field, which is defined in terms of the applied field $E_{0}$. Equation (3) uses the so-called Kemble tunneling formalism (or approximation) [24]. $h, \mu, e, m$, and $k_{B}$ are the Planck constant, Fermi energy, elementary charge, electron mass, and Boltzmann constant, respectively. $B=6.83 \times 10^{9} \mathrm{~V} / \mathrm{m} \mathrm{eV}^{3 / 2}$ is a constant and $v(y)$ is the field-emission elliptic function:

$$
\begin{aligned}
v(y)= & 2^{-1 / 2}\left[1+\left(1-y^{2}\right)^{1 / 2}\right]^{1 / 2} \\
& \times\left(E(k)-\frac{y^{2} K(k)}{1+\left(1-y^{2}\right)^{1 / 2}}\right),
\end{aligned}
$$

where

$$
\begin{aligned}
& y=C E^{1 / 2} / H, \\
& k^{2}=\frac{2\left(1-y^{2}\right)^{1 / 2}}{1+\left(1-y^{2}\right)^{1 / 2}}, \\
& C=3.79 \times 10^{-5} \mathrm{eV} /(\mathrm{V} / \mathrm{m})^{1 / 2},
\end{aligned}
$$

$K(x)$ and $E(x)$ are the complete elliptic integrals of the first and second kind, respectively, and $y$ is the Nordheim parameter.

The current equation [Eq. (1)] is then approximated to be [see Eqs. (1.1a) and (1.1b) in Ref. [22] and Eq. (5) in Ref. [7] with $s=y$ ]

$$
I=\frac{A S E^{2}}{\phi t(y)^{2}} \exp \left(-\frac{B \phi^{3 / 2} v(y)}{E}\right)
$$

with

$$
\begin{aligned}
& y=C E^{1 / 2} / \phi, \quad t(y)=v(y)-\frac{2}{3} y \frac{d v}{d y}, \\
& A=1.54 \times 10^{-6} \\
& \mathrm{~A} \mathrm{eV} /(\mathrm{V} / \mathrm{m})^{2}, \quad B=6.83 \times 10^{9} \mathrm{~V} / \mathrm{meV}^{3 / 2},
\end{aligned}
$$

and $\phi$ being the local work function of the material. The same equation can also be found in Ref. [23] but with the constants $A^{\prime}=1.55 \times 10^{-6} \mathrm{~A} \mathrm{eV} /(\mathrm{V} / \mathrm{m})^{2}$ and $B^{\prime}=$ $6.86 \times 10^{9} \mathrm{~V} / \mathrm{meV}^{3 / 2}$. The difference between $A^{\prime}$ and $B^{\prime}$ and the values in Eq. (6) is negligible and since the values in Eq. (6) are commonly used, we use them in calculating the currents in Fig. 1. 


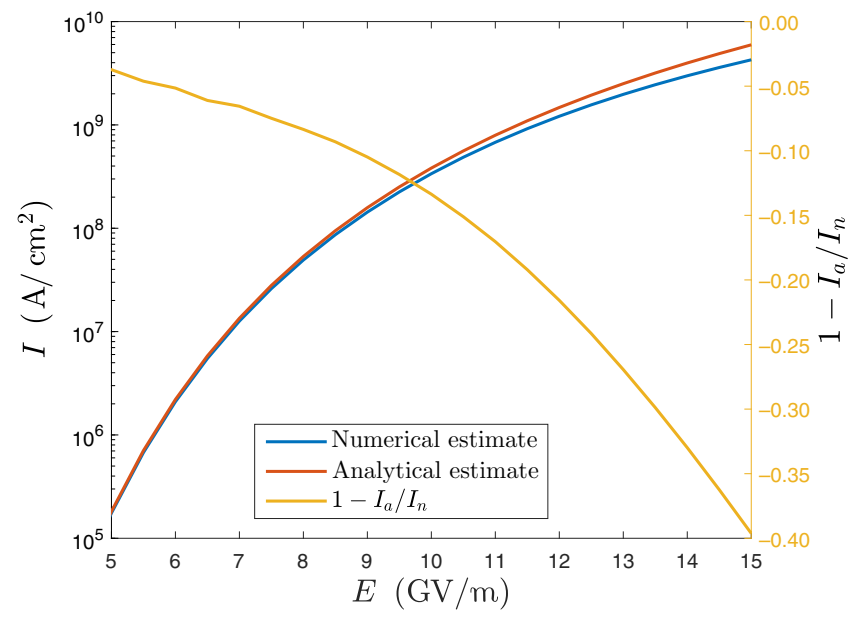

FIG. 1. Comparison between the numerical (blue) and the analytical (red) current estimates of Eq. (5). The deviation between the numerical and the analytical estimates (yellow plot on the right) increases with the field. The error is in the range from approximately $3 \%$ to approximately $40 \%$.

For a more-detailed and general derivation of these equations, see Refs. [11,15].

Equation (5) can be further simplified by approximating $v(y)$ using [25]

$$
v(y) \cong 0.956-1.062 y^{2}
$$

and setting $t(y)=1$ [Eq. (8) in Ref. [7] ]. These assumptions lead to the approximate expression [7]

$$
\frac{d \log _{10}\left(I / E_{0}^{2}\right)}{d\left(1 / E_{0}\right)}=-\frac{G \phi^{3 / 2}}{\beta},
$$

where $G=2.84 \times 10^{9} \mathrm{~V} / \mathrm{meV}^{3 / 2}$, referred to as the "FNplot slope," which allows the commonly used estimation of $\beta[1,17,18,26-28]$. The power of $E$ in the logarithm is 2 in dc systems and 2.5 in rf systems [Eqs. (11) and (14), respectively, in Ref. [7] ].

While this formulation allows a simple analytical evaluation of $\beta$, the validity range of the above approximations is limited. Therefore, in this work, unless stated explicitly that Eq. (8) is being used, the approximations of Eqs. (7) and (8) are not applied, and instead either Eq. (5) or a numerical estimation of Eq. (1) is used with an improved approximation of $v(y)$, presented in the next section.

\section{EXTENDING CURRENT ESTIMATES}

The approximations applied to Eq. (5) leading to Eq. (8) are applicable over a limited range of fields. Applied fields are, in most cases, on the order of $100 \mathrm{MV} / \mathrm{m}$, and thus the analytical approximation provided by Eq. (5), which holds to within $3 \%$, seems reasonable. However, dark current is controlled by local fields in the current-emission region,

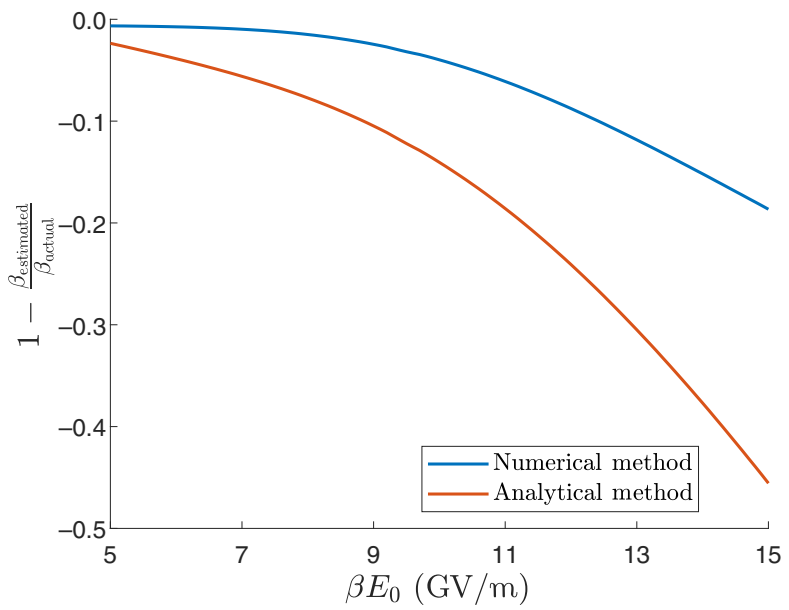

FIG. 2. Comparison between the analytical $\beta$ estimation of Eq. (8) and the numerical method of Eq. (10).

and field enhancement may lead to local fields on the order of up to $10 \mathrm{GV} / \mathrm{m}[1,16-19]$. A comparison of the current as calculated by Eq. (5) - without the approximation of Eq. (7) - with a numerical estimation of Eq. (1) yields significant deviations above $5 \mathrm{GV} / \mathrm{m}$, reaching up to $40 \%$ at fields of $15 \mathrm{GV} / \mathrm{m}$, as demonstrated for copper in Fig. 1. The work function $\phi$ used in Fig. 1 is $4.65 \mathrm{eV}$, and this value is used in the rest of the paper.

In addition, it was previously shown [14] that the approximation provided by Eq. (7) holds to within $10 \%$ for fields above $9 \mathrm{GV} / \mathrm{m}$ in copper. This can be significantly improved with a modified approximation provided by Eq. (9), which leads to an improved fit with an error smaller than $0.33 \%$ for fields up to $15 \mathrm{GV} / \mathrm{m}$ [see Eq. (11) in Ref. [14] with $f=y^{2}$.

$$
v_{\mathrm{F} 06}(y)=1-y^{2}+\frac{1}{3} y^{2} \ln y .
$$

Numerical integration of Eq. (1) using Eqs. (2), (3), (5), and (9) enables the calculation of $\gamma$, the FN-plot slope, which is defined as $\gamma(E)=d \log _{10}\left(I / E_{0}^{2}\right) / d\left(1 / E_{0}\right)$. Furthermore, the local field enhancement $\beta$ can then be estimated using the average field in the range $\tilde{E}_{0}$ :

$$
\beta=\frac{E(\gamma)}{\tilde{E}_{0}} .
$$

The modified equation has an estimation error reduced relative to Eq. (8) by a factor of 2-4 as shown in Fig. 2. The numerical estimation error ranges from about $0.6 \%$ to $18.7 \%$, compared with $2.4 \%$ to $45.6 \%$ for the analytical equation (8).

While there are known issues with the equations used by Wang and Loew (as discussed above), this is still the most commonly used method of $\beta$ estimation, and so we 
use this method as a reference point in this paper. Furthermore, even though there are other implementations of the FN-plot-slope method (e.g., by Houston [23]), these are not expected to be better than the numerical estimates presented in Fig. 2. Both the numerical version of the FNplot-slope method [Eq. (10)] and the data used for the estimation in Fig. 2 are generated by the exact same model. Thus, this implementation provides a best-case scenario for the FN-plot-slope method and circumvents any numerical errors or approximations in the model. Since even this best-case demonstration leads to large errors in higher fields, this indicates that there is an inherent issue with the FN-plot-slope method. This increased deviation at higher fields is consistent with the loss of the linear dependence in the FN plot and can be further studied using the "MG plot" [22]. However, this is not done here.

\section{STATISTICAL ANALYSIS}

\section{A. Applied field-variation}

In experimental systems, the applied fields fluctuate either due to electrical components (e.g., the power supply) or due to rf interference, resulting in a fluctuating current. Normally, the current is averaged and filtered to allow the use of Eq. (8), but these variations can lead to an independent estimation of $\beta$. If we define $\Delta E_{0}$ and $\Delta I$ as the fluctuation amplitude of the applied field and the current density, respectively, the current fluctuation amplitude is given by $\Delta I=\beta(\partial I / \partial E) \Delta E_{0}$. We define

$$
\eta \equiv I^{-1} \frac{\Delta I}{\Delta E_{0}}=\beta I^{-1} \frac{\partial I}{\partial E} .
$$

Equation (11) can be inverted to yield $\beta=\beta\left(\eta, E_{0}\right)$. Using the experimentally measured quantities $I_{m}, \Delta I_{m}$, and $\Delta E_{0 m}$, we calculate $\eta_{m}=I_{m}^{-1}\left(\Delta I_{m} / \Delta E_{0 m}\right)$ and estimate $\beta$ as $\beta_{m}=\beta\left(\eta_{m}, E_{0 m}\right)$. Further details of this method, as well as an alternative representation and an example are provided in Appendix B.

Figure 3 shows the deviation between the estimated $\beta$ $\left(\beta_{\text {estimated }}\right)$ and the value used in the simulation $\left(\beta_{\text {actual }}\right)$ for an applied-field variation with a Gaussian distribution with a standard deviation (SD) of $50 \mathrm{~V} / \mathrm{m}$. As can be seen, $\beta_{\text {estimated }}$ deviates from $\beta_{\text {actual }}$ by up to $3 \%$ for fields $\beta E_{0}$ larger than $7.5 \mathrm{GV} / \mathrm{m}$. This is significantly smaller than the error shown in Fig. 2, where $\beta$ is estimated by the FN-plot-slope method.

In addition, we calculate the $\beta$ estimation error when applied fields are assumed to have a different SD at three average local fields, shown in Fig. 4. As can be seen, increasing the SD of the variation reduces the estimation error up to a certain value, where further increasing the variation increases the error. Around a field variation of $0.5-5 \mathrm{kV} / \mathrm{m}$, the estimation error is a minimum, approximately $0.1 \%$. Thus, $\beta$ estimation can be improved

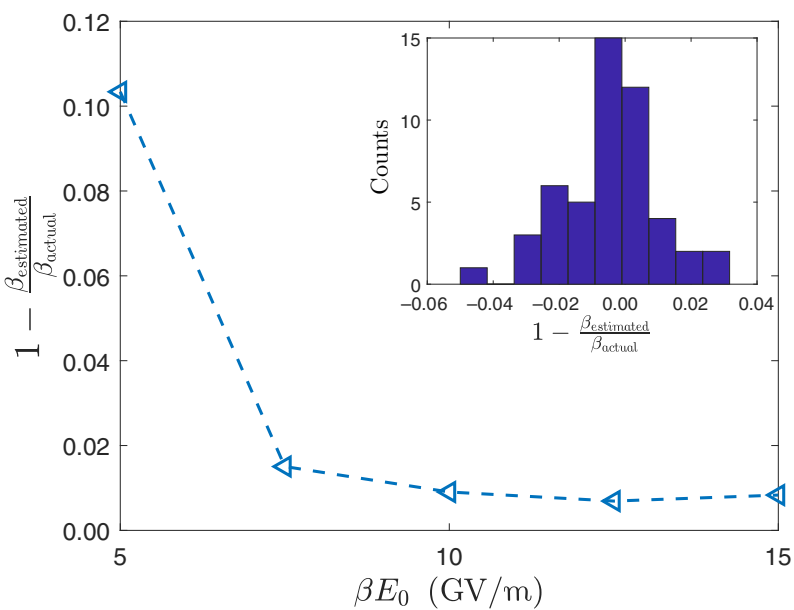

FIG. 3. SD of the relative estimation error of $\beta$ (1$\left.\beta_{\text {estimated }} / \beta_{\text {actual }}\right)$ for the field-variation method. The mean value of the error is approximately zero, with a maximum value of $0.8 \%$. While the error at lower fields (up to about $11 \%$ ) is higher than in Fig. 2, at high fields it is lower- $1 \%-3 \%$ maximum error compared with more than $10 \%$.

if field variation can be controlled appropriately. Possible improvement of the estimation of $\beta$ can also be achieved by use of a non-Gaussian distribution for $E$ in the calculation of $\eta$.

\section{B. Shot noise}

In addition to direct variation due to field intensity, the current varies due to the stochastic nature of tunneling (shot noise). If shot noise indeed controls the current variation, this can be used as a means to probe $\beta$. The amplitude

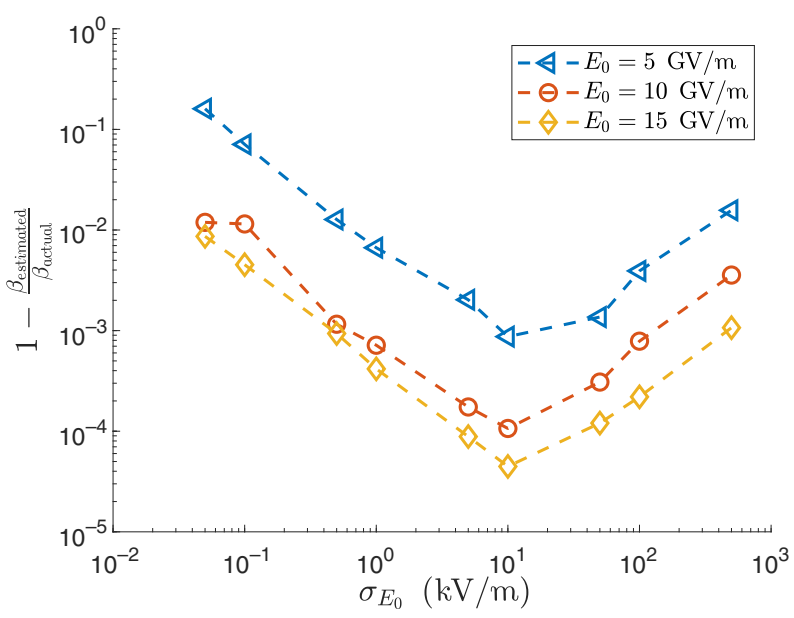

FIG. 4. The field-variation method's expected relative error in estimating $\beta\left(1-\beta_{\text {estimated }} / \beta_{\text {actual }}\right)$ as a function of the amplitude of the local field variation for different mean field values. As can be seen, the error in estimating $\beta$ depends on the field-variation amplitude, with the minimum error achieved around $10 \mathrm{kV} / \mathrm{m}$. 
of the current fluctuations as a function of $\beta$ is evaluated by our modeling the current as a binomial variable with transmission probability $p=D(W)$ and number of trials $n=S N(W) d t d W$ ( $d t$ being the measurement time). Since $S N(W) d t d W \gg 1$ for the relevant parameters of our calculation and energies that contribute the most to the measured current, this is further approximated as a normal distribution with a mean $\tilde{\mu}$ and a SD $\tilde{\sigma}$ given by

$$
\begin{aligned}
& \tilde{\mu}=\operatorname{SdtdWN}(W) D(W), \\
& \tilde{\sigma}^{2}=\operatorname{SdtdWN}(W) D(W)[1-D(W)] .
\end{aligned}
$$

The probability distribution function of the current density $\left[I(W)=S d W J_{W}\right]$ through the emitter is therefore

$P_{W}\left(J_{W}\right) \propto \alpha \exp \left(\frac{\left[J_{W} S d W d t / e-S D(W) N(W) d W d t\right]^{2}}{2 S D(W)(1-D(W)] N(W) d W d t}\right)$,

with $\alpha$ being a normalization factor. Changing from current density $J_{W}$ to the measured current $I(W)$, we get

$$
\begin{aligned}
& P_{I}(I(W)) \\
& \quad=P_{W}\left(J_{W}=\frac{I(W)}{S d W}\right) \\
& \quad \cong \alpha \exp \left(\frac{[I(W)-e D(W) S N(W) d W]^{2}}{2 e^{2} D(W)[1-D(W)] N(W) d W d t^{-1} S^{2}}\right),
\end{aligned}
$$

and so the mean and the variance of the dark current as a function of $W$ are

$$
\begin{aligned}
\mu_{W} & =e S D(W) N(W) d W, \sigma_{W}^{2} \\
& =\frac{e^{2}}{d t} S D(W)[1-D(W)] N(W) d W
\end{aligned}
$$

and the mean and the variance of the total dark current are

$$
\begin{aligned}
& \mu_{I}=I=e S \int D(W) N(W) d W, \\
& \sigma_{I}^{2}=\frac{e^{2}}{d t} S \int D(W)[1-D(W)] N(W) d W .
\end{aligned}
$$

As before, we define a surface area-independent variable:

$$
\theta=1-\frac{d t}{e} \frac{\sigma_{I}^{2}}{I}
$$

In accordance with the method presented in the previous section, $\theta(E)$ is evaluated numerically with Eqs. (1)-(3) and (12) and inverted to generate $E(\theta)$. This is then used to calculate $\beta$, with $\beta=E\left(\theta_{m}\right) / E_{0 m}$, where $\theta_{m}$ is the measured value of $\theta$. A more-detailed explanation of this

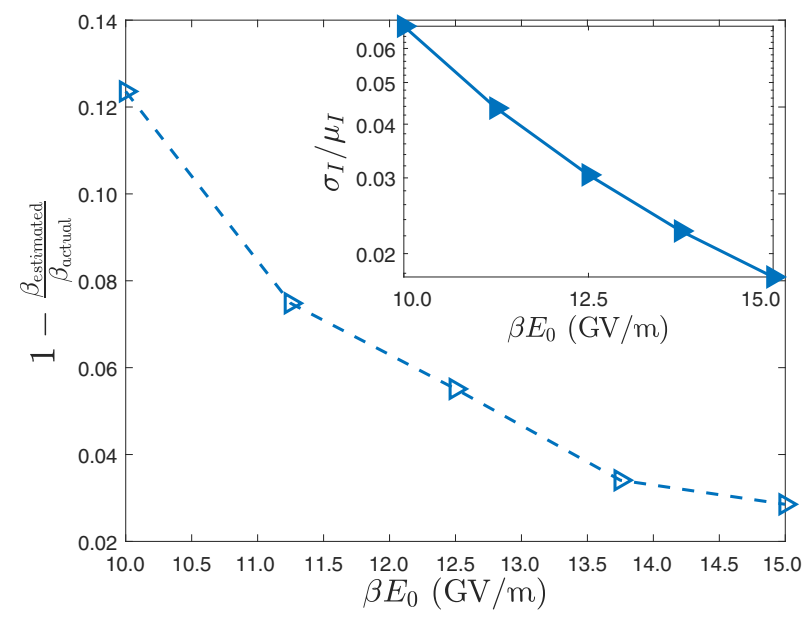

FIG. 5. SD of the shot-noise method's relative error in estimating $\beta\left(1-\beta_{\text {estimated }} / \beta_{\text {actual }}\right)$ as a function of the local field for timescale $d t=1 \mathrm{~ns}$. The inset shows a plot of the ratio $\sigma_{I} / \mu_{I}$ from Eq. (14). For this estimation, it is assumed that field variation is solely due to shot noise.

method is given in Appendix $\mathrm{C}$, along with an explanation for choosing $1-(d t / e)\left(\sigma_{I}^{2} / I\right)$ as opposed to $(d t / e)\left(\sigma_{I}^{2} / I\right)$ and possible corrections that can be made to this method.

Figure 5 shows the deviation between $\beta_{\text {estimated }}$ and the value used in the simulation, $\beta_{\text {actual }}$, for this method. $\beta_{\text {estimated }}$ is calculated with an integration time $d t=1 \mathrm{ns,}$ translating to a measurement bandwidth of $500 \mathrm{MHz}$. As can be seen, the maximum deviation is about $12 \%$ in the range from 10 to $15 \mathrm{GV} / \mathrm{m}$ and the minimum error is about $3 \%$, yielding errors comparable to or smaller than those presented in Fig. 2 in the same range. The results presented in Fig. 5 rely on shot noise being the only source of current variation. Such a condition is not achieved in the experiments described below. In addition, the sampling rate in these experiments is $2.5 \mathrm{kHz}$, which is too slow to allow a useful application of the shot noise-based method, which requires a significantly higher time resolution. While this method has a higher error and smaller validity range (below $10 \mathrm{GV} / \mathrm{m}$ the error becomes as high as $50 \%$ ) than the field-variation method described above, it still has some interest because of the, theoretically, better time resolution it can provide for estimation.

The calculation presented relies on $\theta(E)$ being single valued and monotonous, and for the model presented it is indeed found to be so (see Fig. 9).

It is important to note that the statistical model used here is an approximate one that could be further improved; for example, the number of electrons is assumed to be constant, and further improvements can be made for the calculation of $D(W)$ [Eq. (3)], and noise sources can be added. However, while the actual function of $\theta(E)$ might change somewhat, we expect it to remain single valued and 
monotonous and therefore still useful for the $\beta$-estimation protocol presented here.

Note that we focus on a singular emitter with a single $\beta$ value. In reality, a sample can have multiple emitters with multiple different values of $\beta$ and surface areas (i.e., a nonsingular $S(\beta)$ distribution). It would be pertinent to explore the effect of such distributions of $\beta$ and $S(\beta)$ on the method presented here. The main question would be whether the summation of multiple emitters has a distinct distribution that can be told apart from the distribution for a single emitter, or whether an "effective" emitter would be extracted, thus resulting in an "average" measurement of the emitters.

\section{EXPERIMENTAL RESULTS}

Measurements of dark current versus field are done with two experimental setups: one at CERN, Switzerland, using the dc spark system and the other at Uppsala University, Sweden. Both systems use planar copper electrodes and were built as part of the Compact Linear Collider project for prebreakdown and breakdown studies, with the Uppsala setup extended with cryogenic capabilities [29]. For detailed information about the experimental setups, see Refs. [30,31] for the CERN system and Ref. [29] for the Uppsala University system.

Such measurements results in $I(E)$ plots that vary between experiments even when the same electrodes are used (as can be seen in Fig. 6), and so estimated $\beta$ values also vary. These variations happen even when BD does not occur between measurements. This behavior might result from a number of extrinsic effects (e.g., uncontrolled electrical and environmental modifications), but also may result from intrinsic variation in local $\beta$, which may evolve between and during measurements (e.g., surface evolution). This raises the need for a time-specific and field-specific experimental $\beta$ measurement, as presented here.

Figure 2 shows that the deviation between the estimation equations $(8)$ and $(10)$ increases with increasing local field. The CERN system reaches higher local fields $\left(\beta E_{0}\right)$, and so is the one used to compare these methods. For these data, the voltage is applied continuously, and its value

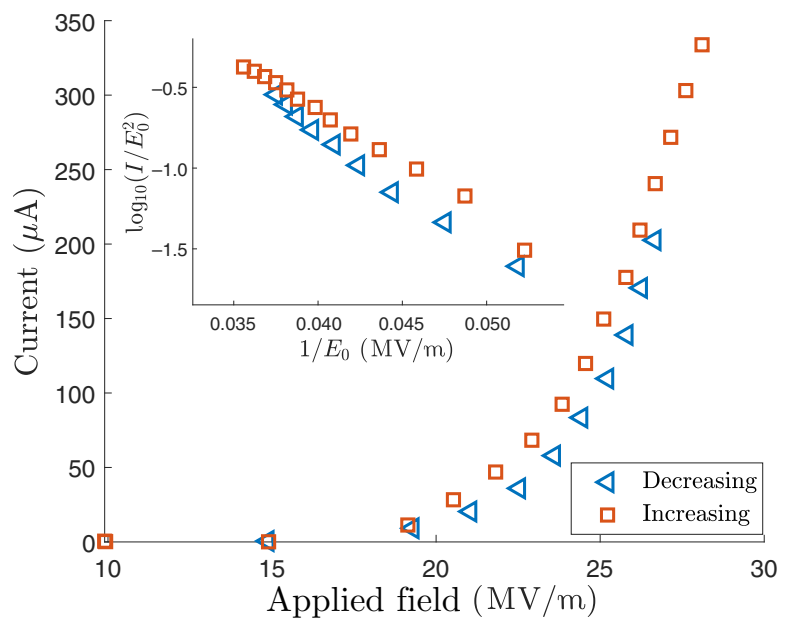

FIG. 6. I-E plot of the analyzed data. The inset shows the FN plot in the range $E_{0}>20 \mathrm{MV} / \mathrm{m}$.

is modified directly through the power supply. The voltage is ramped up until the current reaches a few hundred microamperes and then the procedure is repeated, reversing the voltage polarity. Figure 6 shows the current as a function of the field for the measured data. The measurements analyzed and presented here are all made with the same polarity on the electrodes. Between the decreasing and increasing field profiles, the polarity is swapped for about $10 \mathrm{~min}$, so the two measurements presented, in addition to taking 10-20 min each, are about $10 \mathrm{~min}$ apart.

Table I compares the estimated $\beta$ values for the two slope-analysis methods - Eq. (8) (analytical) and Eq. (10) (numerical) - as well as the measured and expected ratio between the two methods. The $\beta$ estimates based on the slope analysis are dominated by the higher fields, where the semilogarithmic plot of $\log \left(I / E^{2}\right)$ as a function of $1 / E$ is linear (as can be seen in the inset in Fig. 6). The fields used in this analysis are $22-27 \mathrm{MV} / \mathrm{m}$.

For the field-specific estimation, direct estimation of the effect of shot noise on the signal leads to an expected contribution to the current distribution with $\sigma / \mu \sim 0.005$. These fluctuations are small relative to the inherent noise in the measured current, making the shot noise-based estimation impossible. The field variation method also could

TABLE I. Estimated values of $\beta$ for the two FN-plot-slope methods: $\beta_{a}^{\mathrm{FN}}$ is the analytical FN $\beta$ estimate and $\beta_{n}^{\mathrm{FN}}$ is the numerical $\beta$ estimate-Eqs. (8) and (10), respectively. "Up" and "down" refer to an increasing and a decreasing voltage profile, respectively. The applied field range used in the estimations is $22-27 \mathrm{MV} / \mathrm{m}$. The range is chosen so that the FN plot is approximately linear for both increasing and decreasing fields.

\begin{tabular}{|c|c|c|c|c|c|c|}
\hline \multirow[b]{2}{*}{ Method } & \multicolumn{2}{|c|}{$\beta_{\text {estimated }}$} & \multicolumn{2}{|c|}{$\frac{\beta_{a}^{\mathrm{FN}}}{\beta_{\text {estimated }}}$} & \multicolumn{2}{|c|}{ Expected value of $\frac{\beta_{a}^{\mathrm{FN}}}{\beta_{\text {estimatec }}}$} \\
\hline & Up & Down & Up & Down & Up & Down \\
\hline $\begin{array}{l}\beta_{a}^{\mathrm{FN}} \\
\beta_{n}^{\mathrm{FN}}\end{array}$ & $\begin{array}{l}426 \pm 38 \\
391 \pm 63\end{array}$ & $\begin{array}{l}344 \pm 30 \\
323 \pm 52\end{array}$ & $\begin{array}{c}1 \\
1.090\end{array}$ & $\begin{array}{c}1 \\
1.065\end{array}$ & $\begin{array}{c}1 \\
1.089\end{array}$ & $\begin{array}{c}1 \\
1.061\end{array}$ \\
\hline
\end{tabular}


not be applied on the experimental data from the CERN system due to noise but could be performed on the Uppsala University cryogenic data [29]. In this system, the applied voltage is ramped up until either a BD occurs or a current limit is reached. In the data presented, the current limit is reached. For measurements at 30 and $60 \mathrm{~K}$, both the FN-plot-slope method and the field-variation method are used [Eqs. (8) and (11)]. The field statistics are of a "sawtooth" (ramping) form, with the data being divided into bands of $d E_{0} \cong 1.3 \mathrm{MV} / \mathrm{m}(100 \mathrm{~V})$, which is about $2.3 \%$ of the maximum applied field. For each band, ten points are chosen for the analysis, evenly spaced in the voltage. The difference between the field-specific $\beta$ and the "average" FN-plot-slope $\beta$ is about $10 \%-18 \%$, with the FN-plot-slope estimation being higher. Figure 7 shows an example of the field-specific estimation at $30 \mathrm{~K}$. While there does seem to be an apparent trend in $\beta$, the small number of points used for the estimation (limited by the measurement frequency) results in high estimated errors [32], which makes any apparent trend unreliable.

One potential reason that the field-specific $\beta$ is lower than the FN-plot-slope $\beta$ is that in this measurement greater noise is measured for the current compared with the applied field. From Eq. (11) we see that greater current noise, compared with the field noise, results in a higher estimated $\eta$, which, on the basis of the slope of $\eta\left(\beta, E_{0}\right)$ (see Fig. 8), results in a lower $\beta$ estimation for a given $E_{0}$ value.

\section{DISCUSSION}

Current $\beta$ estimation, which is based on the FN-plot slope, requires a wide range of fields, regardless of the mathematical analysis method used to extract the $\beta$ value (numerical or analytical). Precise evaluation of this slope

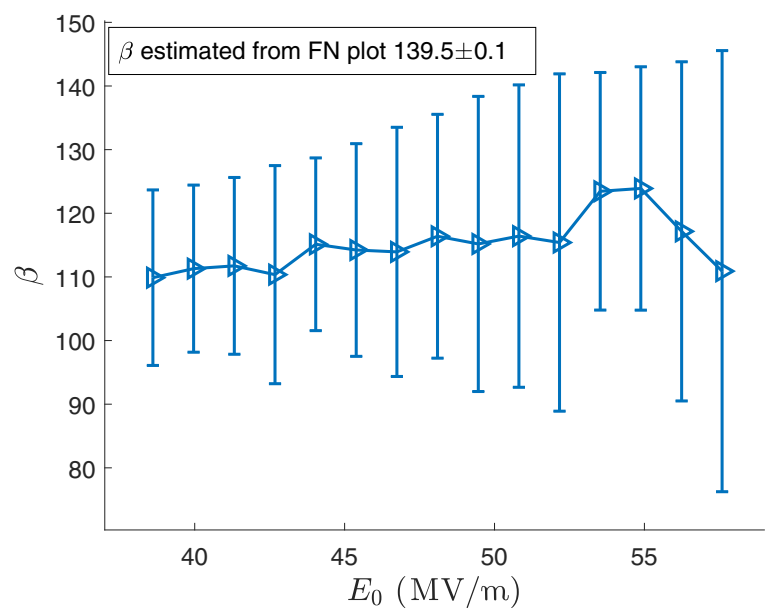

FIG. 7. Example of field-specific $\beta$ estimation at $30 \mathrm{~K}$ with the field-variation method. The estimated $\beta$ value obtained with the FN-plot-slope method of Eq. (8) is $139.5 \pm 0.1$.

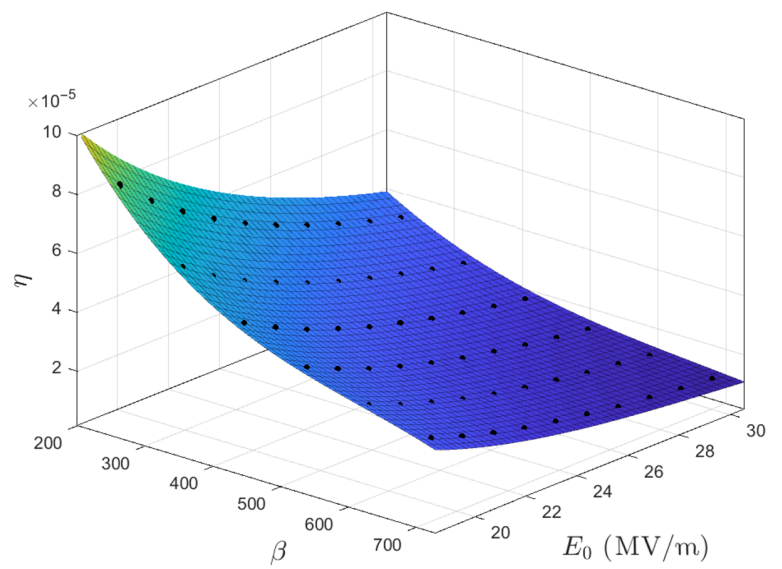

FIG. 8. An example of the calculated values of $\eta$ in the parameter space $\beta \in[240,700]$ and $E_{0} \in[19,30] \mathrm{MV} / \mathrm{m}$ as well as the fit of these points to the polynomial that is used for the estimation. The black points are the calculated values of $\eta$ and the surface is the fit to these points. The material is copper with work function $\phi=4.65 \mathrm{eV}$.

requires long measurement times. These long times allow surface evolution during and between measurements, as well as variation of other hidden system parameters [33]. This may lead to the observed variation in the $\beta$ estimation reported in Table I. In addition, the analysis is dominated by the currents measured at higher fields, where the estimation error is higher (Fig. 2). This last contribution is reduced when the numerical evaluation is applied, as demonstrated by the reduced variation in $\beta_{a}^{\mathrm{FN}}$ and $\beta_{n}^{\mathrm{FN}}$ in Table I.

The experimental methods presented in this paper provide the ability to measure $\beta$ as a function of the applied field and with a much shorter measurement time. This would allow the characterization of $\beta$ in a field, which is currently assumed to be constant but has yet to be verified experimentally.

This is shown experimentally in Fig. 7. The deviation between the FN-plot-slope estimation and the field-specific estimation is between $10 \%$ and $18 \%$, some of which is explained by the model used not accounting for noise. This can be corrected in future work. The estimated errors are too large for us to confidently say anything about the trend of $\beta$ as a function of the field, and this can be solved by using a higher sampling rate in the future.

The field-specific methods presented here could also prove to be more efficient for the characterization of highfield systems since if $\beta$ does vary with the field, it is the high-field $\beta$ that impacts performance and is therefore of more interest. These methods allow the direct estimation of $\beta$ in the range of interest with increased precision (Figs. 3 and 5).

In addition to field-dependent variation, $\beta$ may also evolve dynamically (as a function of time) due to structural 


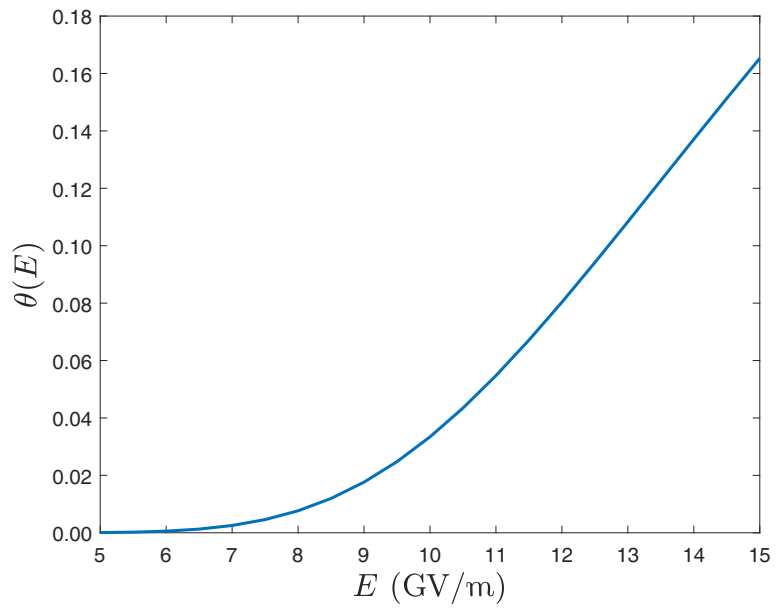

FIG. 9. Plot of $\theta(E)$ for copper with work function $\phi=$ $4.65 \mathrm{eV}$.

evolution of the sample $[8,33]$. The suggested instantaneous methods allow the measurement of such evolution, possibly even during operation, which the FN-plot-slope method does not. The dynamic evolution of $\beta$, if it exists, can be directly related to the BD nucleation process.

With regard to the field-variation method, different statistics and amplitudes for the field variation may result in different errors. For example, for a Gaussian field variation, the optimal amplitude for the field variation is around $1 \mathrm{kV} / \mathrm{m}$ (Fig. 4). Future work could also explore the benefit of using a non-Gaussian distribution of fields, and their respective optimal variation amplitude, to create a faster and more-precise estimate. Appendix A details how to implement the field-variation estimation method with a sinusoidal, a sawtooth, or a Gaussian field profile. The code can be found in Ref. [34]. To apply the shot noise-based method, a combination of small emitters and high sampling rates is required (for Fig. 5 we use 500 $\mathrm{MHz}$ ).

In conclusion, we present two methods that allow a localized, field-dependent $\beta$ estimation. These methods show expected increased accuracy over the commonly used method and allow faster, field-dependent estimation. One of these methods is shown experimentally to be in good agreement with current methods.

\section{ACKNOWLEDGMENTS}

S.L. thanks Dr. Richard G. Forbes for his contribution and guidance with this paper. S.L. and Y.A. acknowledge funding from the PAZY Foundation.

\section{APPENDIX A: FIELD-VARIATION IMPLEMENTATION}

This appendix details how to implement the fieldvariation estimation with the code provided in Ref. [34].
(1) Determine the field-variation statistics. The code provided can handle a sinusoidal or a sawtooth wave function (both can be applied by the power supply) or a Gaussian distribution of fields (as presented in this paper).

(2) Identify the statistical parameters. For the Gaussian case identify the SD of the variation in $E_{0}$ (assumed to be constant in a field). For the sinusoidal and sawtooth cases, identify the magnitude of the function. For all three, identify the number of points used for the estimation and the integration time for each current measurement.

(3) Set the expected range of $\beta$ values. This range could be as large as you like, although local fields of $\beta E_{0} \lesssim$ $3.6 \mathrm{GV} / \mathrm{m}$ tend to yield noisy data.

(4) Run the supplied function "createVarEref" with the above data and save the supplied ".mat" file. A larger $\beta$ range would make this step longer to run but the actual estimation time would not be impacted.

(5) With this saved file, you can now perform several measurements of the mean current $I_{i}$ and the mean applied field $E_{0, i}$. Calculate the SD of the applied field, the measured current, and the mean measured current $\left[I_{m}=\right.$ $(1 / N) \sum_{i=1}^{N} I_{i}$ in Eq. (11)].

(6) Use the supplied function "varEestimation" with the measured parameters from the previous step to get an estimation of $\beta$.

In step 2, for the sinusoidal and sawtooth cases it is important to note that it is assumed the points are sampled uniformly in the cycle of the functions, although it is not assumed that the measurement starts at a particular phase of the function, rather it is assumed that the phase is random.

\section{APPENDIX B: APPLIED-FIELD-VARIATION ALGORITHM}

The applied-field-variation algorithm relies on the variation of the measured mean current, $\Delta I$, as a result of a variation of the applied field $\Delta E_{0}$. This variation is given by

$$
\Delta I=\frac{\partial I}{\partial E} \Delta E=\frac{\partial I}{\partial E} \Delta E_{0} \beta .
$$

Since the current (and its variation) is linearly dependent on the unknown tunneling surface [S in Eq. (5)], we cannot use this single equation to calculate the two unknown variables, $\beta$ and $S$. To address this, we normalize this variation by the mean current $I$, which yields

$$
\eta \equiv \frac{1}{I} \frac{\Delta I}{\Delta E_{0}}=\frac{\beta}{I} \frac{\partial I}{\partial E}=\frac{1}{I} \frac{\partial I\left(\beta E_{0}\right)}{\partial E_{0}} .
$$

This quantity is independent of $S$ and is a function of only measurable quantities and $\beta$, and can therefore be used to estimate $\beta$. 
For a set of measured values $I_{m}, \Delta I_{m}$, and $\Delta E_{0 m}$ we can calculate $\eta_{m}$, and solve the equation

$$
\eta_{m}=\frac{\beta}{I} \frac{\partial I}{\partial E}
$$

using the analytical MG current equation [Eq. (5) and the first equation in Ref. [23]] with the proper approximation for $v(y)$ [Eq. (9)]. This approach is not as effective as the one presented below. This is due to the discrepancy between the analytically and numerically estimated currents (Fig. 1). This estimation equation can also be calculated with other analytical solutions, which may yield better results but are not the focus of this paper.

The method we implement and present in this paper (and the code that is provided in Ref. [34]) uses a numerically generated approximation of the function $\eta\left(\beta, E_{0}\right)$, and together with a given measured $\eta_{m}$ and $E_{0 m}$ calculates the corresponding $\beta$ value with the inverse of that function, $\beta\left(\eta, E_{0}\right)$. For this approach, we assume a range of fields for $E_{0}$ (supplied by the conditions of the experimental setup) and $\beta$ values (chosen around the initial $\beta$ estimation achieved by the FN-plot-slope estimation method and then adjusted according to the results of the estimation). Additionally, a statistical model for the field variation must be assumed. As stated in the main text, the code provided can handle Gaussian, sinusoidal or sawtooth (linear ramping) variations of the field. Once the statistical model has been determined, the variation magnitude must also be measured (the SD of the Gaussian or the peak-to-peak amplitude of the sine and/or sawtooth).

With these parameters, we sample the parameter space of $\eta\left(\beta, E_{0}\right)$ at linearly spaced points. With these points of $\eta\left(\beta, E_{0}\right)$ in the given range, the data are fit to a polynomial. The polynomial is of a maximum order of 5 in both terms and a maximum order of 3 in $\beta$; that is, $\eta\left(\beta, E_{0}\right) \cong$ $\sum_{i j} a_{i j} \beta^{i} E_{0}^{j}$, with $i \leq 3, i+j \leq 5$, and $a_{i j}$ the fitting coefficient. An example of the data and fit is seen in Fig. 8. This fit is injective with regard to $\beta$ for any given $E_{0}$ value in the supplied range for parameters that satisfy $5 \mathrm{GV} / \mathrm{m}<\beta E_{0}<15 \mathrm{GV} / \mathrm{m}$. Values outside this range are not tested as this is the field range of interest here (as explained in Sec. I). With this fit for $\eta\left(\beta, E_{0}\right)$, the value of $\beta$ can be estimated by solving the polynomial equation with the measured parameters of $\eta_{m}$ and $E_{0 m}$ and selecting the value within the supplied $E_{0}$ range (in general there are three solutions for this third-degree polynomial, of which only one is in the desired range). The estimation equation (solving for $\beta$ ) is written explicitly as

$$
\eta_{m}=\sum_{i j} a_{i j} \beta^{i} E_{0 m}^{j} ; \quad i \leq 3, i+j \leq 5
$$

\section{APPENDIX C: SHOT-NOISE ALGORITHM}

The shot-noise algorithm relies on the fact that the current is statistical in nature and that the statistics are a function of the local field. Following the same equations as presented in Sec. IV B, we eventually arrive at the mean and variance of the measured current [Eq. (14)]:

$$
\begin{aligned}
& \mu_{I}=I=e S \int D(W) N(W) d W \\
& \sigma_{I}^{2}=\frac{e^{2}}{d t} S \int D(W)[1-D(W)] N(W) d W
\end{aligned}
$$

Now we can define a new parameter that is independent of $S$ :

$$
\theta(E)=1-\frac{d t}{e} \frac{\sigma_{I}^{2}}{\mu_{I}}
$$

A plot of $\theta(E)$ is shown for copper in Fig. 9. The reason we use this formulation instead of simply using $(d t / e)\left(\sigma_{I}^{2} / \mu_{I}\right)$ is that in the energy-specific form

$$
\begin{aligned}
\frac{d t}{e} \frac{\sigma_{I}^{2}}{\mu_{I}} & =\frac{d t}{e} \frac{\frac{e^{2}}{d t} S \int D(W)[1-D(W)] N(W) d W}{e S \int D(W) N(W) d W} \\
& =1-D(W, E)
\end{aligned}
$$

and so this way of defining $\theta$ has some connection to the physical quantity $D(W, E)$, the transmission probability, which is directly dependent on the desired $\beta$. It is also worth noting that $\theta(E)$ is independent of $d t$.

We can use the inverse function $E(\theta)$ and a measurement of $\theta_{m}$ to get a direct estimation of the local field $E_{m}=E\left(\theta_{m}\right)$ and the field enhancement factor:

$$
\beta_{m}=\frac{E_{m}}{E_{0 m}}=\frac{E\left(\theta_{m}\right)}{E_{0 m}}
$$

We can see from Fig. 9 that for a given estimation error in $\theta$, the estimation error in $E$ is largest at lower fields due to the slope of the graph.

For the implementation presented in this paper, the $\theta(E)$ plot is generated as shown above and the fitting is done for $E^{1.8}\left(\theta^{0.81}\right)$, as this is found to yield slightly better results. The optimization of this method is not the focus of this paper, so it is possible a better method can be used to yield better results than those presented here.

This method can be improved upon in several ways:

(1) Improvements to the $N(W)$ and $D(W, E)$ functions.

(2) Improvements to the statistical model. The model used here is a binomial distribution approximated to be Gaussian. This implies a nonstatistical incident electron current and can also be expanded. 
(3) Adding noise sources. Noise is completely ignored in this paper. In actual systems noise can be a significant factor, and accounting for it could help increase the accuracy of the method.

(4) Accounting for multiple emitters. We assume here an emitter with a single $\beta$ and effective emitting area $S$. However, electrodes are expected to have multiple emitters, each with its own field enhancement factor and area. Future research into the impact of a nonsingular surface area distribution $S(\beta)$ could help understand whether a collection of emitters behaves qualitatively different from an "effective" emitter (with $\beta_{\text {eff }}$ and $S_{\text {eff }}$ ) or whether they cannot be told apart.

[1] A. Descoeudres, Y. Levinsen, S. Calatroni, M. Taborelli, and $\mathrm{W}$. Wuensch, Investigation of the dc vacuum breakdown mechanism, Phys. Rev. Accel. Beams 12, 092001 (2009).

[2] M. Veske, S. Parviainen, V. Zadin, A. Aabloo, and F. Djurabekova, Electrodynamics — molecular dynamics simulations of the stability of $\mathrm{Cu}$ nanotips under high electric field, J. Phys. D 49, 215301 (2016).

[3] S. Parviainen, F. Djurabekova, H. Timko, and K. Nordlunds, Electronic processes in molecular dynamics simulations of nanoscale metal tips under electric fields, Comput. Mater. Sci. 50, 2075 (2011).

[4] A. Kyritsakis and F. Djurabekova, A general computational method for electron emission and thermal effects in field emitting nanotips, Comput. Mater. Sci. 128, 15 (2017).

[5] G. A. Mesyats, Explosive processes on the cathode in a vacuum discharge, IEEE Trans. Electr. Insul. EI-18, 218 (1983).

[6] G. A. Loew and J. W. Wang, International symposium on discharges and electrical insulation in vacuum (SLAC-PUB, Santa Fe, NM, United States, 1990), https://www.osti.gov/biblio/6488947.

[7] J. W. Wang and G. A. Loew, Field emission and rf breakdown in high-gradient room temperature linac structures. Technical Report 7684, SLAC PUB, Oct 1997.

[8] E. Z. Engelberg, J. Paszkiewicz, R. Peacock, S. Lachmann, Y. Ashkenazy, and W. Wuensch, Dark current spikes as an indicator of mobile dislocation dynamics under intense dc electric fields, Phys. Rev. Accel. Beams 23, 123501 (2020).

[9] A. D. Cahill, J. B. Rosenzweig, V. A. Dolgashev, S. G. Tantawi, and S. Weathersby, High gradient experiments with $x$-band cryogenic copper accelerating cavities, Phys. Rev. Accel. Beams 21, 102002 (2018).

[10] E. L. Murphy and R. H. Good, Thermionic emission, field emission, and the transition region, Phys. Rev. 102, 1464 (1956).

[11] R. G. Forbes and J. H. B. Deane, Reformulation of the standard theory of Fowler-Nordheim tunnelling and cold field electron emission, Proc. R. Soc. A 463, 2907 (2007).

[12] R. H. Fowler and L. Nordheim, Electron emission in intense electric fields, Proc. R. Soc. A 781, 173 (1928).
[13] W. W. Dolan, Current density tables for field emission theory, Phys. Rev. 91, 510 (1953).

[14] R. G. Forbes, Simple good approximations for the special elliptic functions in standard Fowler-Nordheim tunneling theory for a Schottky-Nordheim barrier, Appl. Phys. Lett. 89, 113122 (2006).

[15] R. G. Forbes, Fowler-Nordheim plot analysis: A progress report, Jordan J. Phys. 8, 125 (2015).

[16] P. Kranjec and L. Ruby, Test of the critical theory of electrical breakdown in vacuum, J. Vac. Sci. Technol. 4, 94 (1967).

[17] S. V. Baryshev, S. Antipov, J. Shao, C. Jing, K. J. Pérez Quintero, J. Qiu, W. Liu, W. Gai, A. D. Kanareykin, and A. V. Sumant, Planar ultrananocrystalline diamond field emitter in accelerator radio frequency electron injector: Performance metrics, Appl. Phys. Lett. 105, 203505 (2014).

[18] H. Timko, K. Ness Sjobak, L. Mether, S. Calatroni, F. Djurabekova, K. Matyash, K. Nordlund, R. Schneider, and W. Wuensch, From field emission to vacuum arc ignition: A new tool for simulating copper vacuum arcs, Contrib. Plasma Phys. 55, 299 (2015).

[19] R. Zennaro, H. Blumer, M. Bopp, T. Garvey, L. Rivkin, N. Catalan-Lasheras, A. Grudiev, G. McMonagle, A. Solodko, I. Syratchev, R. Wegner, B. Woolley, W. Wuensch, T. G. Lucas, M. Volpi, T. Argyropoulos, and D. Esperante-Pereira, in Proceedings of International Particle Accelerator Conference (IPAC'17), Copenhagen, Denmark, May, 2017, number 8 in International Particle Accelerator Conference, p. 4318, Geneva, Switzerland, May (JACoW, Geneva, Switzerland, 2017), https://doi.org/10.18429/JACoW-IPAC2017-THPIK097.

[20] R. G. Forbes, J. H. B. Deane, A. Fischer, and M. S. Mousa, Fowler-nordheim plot analysis: a progress report, Jordan J. Phys. 8, 125 (2015).

[21] Richard G. Forbes, in 2018 31st International Vacuum Nanoelectronics Conference (IVNC) (IEEE, Kyoto, Japan, 2018), p. 126.

[22] R. G. Forbes, The murphy-good plot: A better method of analysing field emission data, R. Soc. Open Sci. 6, 190912 (2019).

[23] J. M. Houston, The slope of logarithmic plots of the Fowler-Nordheim equation, Phys. Rev. 88, 349 (1952).

[24] Edwin C. Kemble, A contribution to the theory of the B. W. K. method, Phys. Rev. 48, 549 (1935).

[25] F. M. Charbonnier and E. E. Martin, A simple method for deriving, from measured $I(V)$ data, information on the geometry of a field emission current source of unknown characteristics, J. Appl. Phys. 33, 1897 (1962).

[26] M. Kildemo, New spark-test device for material characterization, Nucl. Instrum. Methods Phys. Res. B 530, 596 (2004).

[27] M. Radmilovic-Radjenovic and B. Radjenovic, A particlein-cell simulation of the high-field effect in devices with micrometer gaps, IEEE Plasma Sci. 35, 1223 (2007).

[28] P. Piot, C. A. Brau, B. K. Choi, B. Blomberg, W. E. Gabella, B. Ivanov, J. Jarvis, M. H. Mendenhall, D. Mihalcea, H. Panuganti, P. Prieto, and J. Reid, Operation of an ungated diamond field-emission array cathode in a $L$-band radio frequency electron source, Appl. Phys. Lett. 104, 263504 (2014). 
[29] M. Jacewicz, J. Eriksson, R. Ruber, S. Calatroni, I. Profatilova, and W. Wuensch, Temperature-Dependent Field Emission and Breakdown Measurements Using a Pulsed High-Voltage Cryosystem, Phys. Rev. Appl. 14, 061002 (2020).

[30] N. C. Shipman, PhD thesis, University of Manchester, 2014.

[31] A. Korsback, F. Djurabekova, L. Mercadé Morales, I. Profatilova, E. Rodriguez Castro, W. Wuensch, S. Calatroni, and T. Ahlgren, Vacuum electrical breakdown conditioning study in a parallel plate electrode pulsed DC system, Phys. Rev. Accel. Beams 23, 033102 (2020).
[32] The estimated error in $\beta$ is $\Delta \beta=|E(\eta)-E(\eta \pm \Delta \eta)| E_{0}^{-1}$, with $\Delta \eta=\sqrt{(\partial \eta / \partial I)^{2} \delta I_{m}^{2}+\left(\partial \eta / \partial \sigma_{I}\right)^{2} \delta \sigma_{I_{m}}^{2}+\left(\partial \eta / \partial \sigma_{E_{0}}\right)^{2} \delta \sigma_{E_{0}}^{2}}$ the estimated error in $\eta . \delta I_{m}, \delta \sigma_{I_{m}}$, and $\delta \sigma_{E_{0} m}$ are the estimated errors in the measurement of the mean current and the current and field fluctuations, respectively.

[33] E. Z. Engelberg, Y. Ashkenazy, and M. Assaf, Stochastic Model of Breakdown Nucleation under Intense Electric Fields, Phys. Rev. Lett. 120, 124801 (2018).

[34] S. Lachmann, Source code for field variation estimation. https://github.com/SagyLachmann/Statistical-analysis-ofdark-currents, (2021). [Online]. 